\title{
Manufacture and characterization of three- component nano-composites Hydroxyapatite Using Polarization Method
}

\author{
Khalili,Alireza $^{* 1}$, Naeimi, Farid ${ }^{2}$, Rostamian,Mostafa ${ }^{3}$, baradaran Shoraka,Zahra ${ }^{4}$, Tohidi, Shabnam ${ }^{5}$ \\ ${ }^{1}$ :Materials Engineering Department,South Tehran Branch,Islamic Azad University,Tehran, Iran. \\ ${ }^{2}$ :Advanced Materials Research Center, Materials Engineering Department, Najafabad, Iran \\ ${ }^{3}$ :Department of Biomedical Engineering, South Tehran Branch, Islamic Azad University,Tehran, Iran. \\ ${ }^{4}$ :Biomechanic Engineering Department, Islamic Azad University, Mashhad, Iran. \\ 5: Faculty of Materials Science and Engineering, K. N. Toosi University of Technology, Tehran, Iran
}

\begin{abstract}
The current study aimed to manufacture and characterization of three-component nanocomposites Hydroxyapatite Using Polarization Method.. The new work in this paper is an antibacterial test for triple coating which reduces the number of bacteria perceptibly. the antibacterial and corrosion behaviors were evaluated in comparison to uncoated samples by the electrochemical coating polarization test method. Tests such as $\mathrm{X}$-ray diffraction and scanning electron microscope were used to characterize the coating. The $X$-ray diffraction test results showed that a three-component composite coating was created on the substrate in optimal coating conditions. The scanning electron microscope images revealed that the morphology of the formed coating surface was uniform, and its thickness was about 22 to 26.5 micrometers. The results suggested that coating thickness increased with the progression of coating time. Corrosion test outcomes indicated that employing the nanocomposite layer enhanced the corrosion resistance of the coating. Antibacterial test results suggested that the application of the nanocomposite coating effectively reduced the bacteria growth on the surface.
\end{abstract}

Keywords: Nanocomposite coating, Antibacterial, polarization method, Hydroxyapatite

\section{Introduction}

High demand and the increasing use of implants have led to notable improvements in biomaterial field; therefore, many studies have been conducted in this area in recent years [1]. One of the essential characteristics of a biomaterial is its biocompatibility. In other words, these materials shouldn't be toxic or carcinogenic, and they shouldn't have an adverse reaction with fluid material within the body. They must have mechanical robustness and long fatigue life and proper density [2].

The characteristics of Titanium and its alloys involve low density, high stability, high corrosion resistance, low module, high biocompatibility, complete neutrality to the body, and high capacity to form a connection with bones and other tissues. Owing to these properties, they are attracting attention, especially in medicine [3-4]. However, relatively poor bioactivity joined with inapt attachment to the body tissue has led to the suppression of this alloy in the human body. One of the typical approaches to improve bioactivity behavior is to employ Hydroxyapatite coating.

Due to the high similarity to human bone in terms of biology and chemical structure as well as desired biocompatibility, Hydroxyapatite- and especially Calcium hydroxyapatite- is one of the most broadly used and suitable calcium phosphate ceramics [5]. The word "Apatite" consists of a group of compounds that are identical in structure but do not have the same chemical compositions necessarily. Calcium hydroxyapatite with the chemical formula of $\mathrm{Ca}_{10}\left(\mathrm{Po}_{4}\right)_{6} \mathrm{OH}_{2}$ has a hexagonal geometry. Hydroxyapatite has an appealing and accepting structure letting many ions to be settled within it [6].

One of the latest composites includes using Graphene oxide and zirconium oxide with apatite hydroxide as the coating for implant materials. Zirconia's suitable corrosion resistance has been appealing mainly in forming the oxide film. The oxide film, with its surrounding tissue, does not display any biological or chemical bonds at the implant. Through some studies, scientists have discovered that another approach to improve Zirconia's corrosion resistance and biocompatibility is creating composite films of Zirconia/Hydroxyapatite [7]. Graphene's exceptional properties include high current density, ballistic shipping, chemical inertness, high thermal conductivity, optical transmittance, and ultra-hydrophobicity [8]. Graphene also exhibits high-grade covalent and non-covalent functionalization capability with various materials resulting in its dissolution in different solvents [9]. 
The graphene oxide, zirconium oxide, and hydroxyapatite compounds are applied by various methods. These techniques involve cold spray, dip coating, flame spray, and electrophoretic methods. Due to some factors, including the lower cost, low process temperature, and proper process control, prefer the electrophoretic method to others. The results obtained from different studies suggested that these types of amplifiers can cause problems and disorders.

One of the problems is the absorption of germs. Natural products are still of the chief sources of today's pharmaceutical molecules that are derived from prokaryotic bacteria, eukaryotic microorganisms, various plants and organisms, and animals. Microbial and herbal products make up the largest portion of the antimicrobial material ever discovered [10].

In a similar study, Pen et al. [11] successfully precipitated hydroxyapatite/graphene oxide/silk nanocomposite coats on a titanium substrate using the electrophoretic method. Graphene and hydroxyapatite nanoparticles are distributed uniformly in the Silk matrix, which has a dense and smooth topography. Comparing the pure silk with the ternary compound, it was observed that mechanical properties have been improved. In a similar study, Ana Jankovich et al. [12] successfully covered a titanium layer with a graphene/ hydroxyapatite composite coating. Scanning Electron Microscope images of the coating indicated that the cracks are suddenly lesser than the situation where the coating was hydroxyapatite only. In another study, Farnoosh et al. [13] investigated the effect of stability of nano-hydroxyapatite/Zirconia powder suspension stabilized with 0/10/20/40 specific values of nano-zirconia powder on the structure, strength of bonds, and electrochemical behavior in a simulated body solution using the electrophoretic method on a titanium alloy substrate. According to the Zeta potential theory, the highest clotblocking energy in this study was observed in the H20Z sample (19.93). The authors also decided that the highest zeta potential (38.08) of the suspension contains $20 \%$ zirconia in $0.19 \mathrm{~g}$ iodine. In another study, Ming et al. [14] comprehensively summarized the research fundamentals and development in graphene-based materials of electrophoretic over the last five years. Comprehensiveness and effectiveness of the electrophoretic method to produce graphene and composite materials for a variety of applications, especially in the electrophoretic process, had been investigated in that research. This study aimed to characterize the nanocomposite coating of hydroxyapatite, zirconia, and graphene oxide on a titanium substrate using the polarization method, and investigate its antibacterial behavior.

\section{Materials and methods}

The $\mathrm{Ti}_{6} \mathrm{Al}_{4} \mathrm{~V}$ alloy was employed in this study as a widely used medical biomaterial. The dimensions of the used sample have been presented in Figure (1). The samples were sanded by sandpapers with silicon carbide abrasive particles at 400-600-800-1200 for grinding operations, respectively. For degreasing, the samples were dipped in distilled water and were put in the ultrasonic apparatus for thirty minutes, and were finally rinsed with Aston.

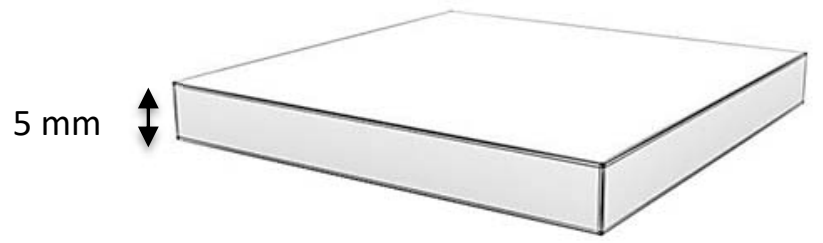

Figure 1. Schematic of the sample's dimensions

Table 1. Characteristics of the Ti-6AL-4V alloy used in this study

\begin{tabular}{|c|c|c|c|c|c|c|c|}
\hline Element & V & Al & Fe & O & C & N & He \\
\hline Percentage & 4.5 & 4.5 & 3 & 2 & 0.8 & 0.5 & 0.15 \\
\hline
\end{tabular}

In this study, the Hydroxyapatite powder produced by Sigma Aldrich USA was used below $200 \mathrm{~nm}$. This powder's characteristics have been included in Table (2).

Table 2. Characteristics of the Hydroxyapatite powder alloy used in this study

\begin{tabular}{|c|c|c|}
\hline Chemical formula & Molecular weight $(\mathbf{g} / \mathbf{m o l})$ & Material name \\
\hline $\mathrm{Ca}_{10}\left(\mathrm{po}_{4}\right)_{6} \mathrm{OH}_{2}$ & $502 / 31$ & Hydroxyapatite \\
\hline
\end{tabular}

Also, Zirconia oxide powder, manufactured by Merck German Company, was employed at the dimensions below $100 \mathrm{~nm}$. 
Table 3. Characteristics of the Zirconia oxide powder alloy used in this study

\begin{tabular}{|c|c|c|}
\hline Chemical formula & Molecular weight $\mathbf{( g / m o l})$ & Material name \\
\hline $\mathrm{Zro}_{2}$ & $123 / 21$ & Zirconia oxide \\
\hline
\end{tabular}

Moreover, in this study, Graphene oxide was produced by Sigma Aldrich USA at dimensions below $100 \mathrm{~nm}$. The characteristics of the used powder have been presented in Table (4).

Table 4. Characteristics of Graphene oxide powder alloy used in this study

\begin{tabular}{|c|c|c|}
\hline Chemical formula & Molecular weight $\mathbf{( g / \mathbf { m o l } )}$ & Material name \\
\hline $\mathrm{GO}$ & $120 / 52$ & Graphene oxide \\
\hline
\end{tabular}

In this study, the selected composite powder contained Hydroxyapatite with graphene oxide and zirconium oxide, the production procedure and characteristics of which, were described. Specific and optimum amounts of Hydroxyapatite, graphene oxide and zirconium oxide were mixed with $50 \mathrm{~mL}$ of isopropanol from Merck Germany. The mixture was then put in a magnetic stirrer. To dissolve better and create a positive charge on the surface of the hydroxyapatite particles, other than increasing the stability of the suspension, and moving of the particle toward the cathode and their deposition on its surface in the electrophoretic process, the specified amount of Merck's triethanolamine was added. Then, the mixture was on the stirrer for 48 hours. Moreover, with the help of Merck's nitric acid, the $\mathrm{pH}$ of the suspension was reduced to about 3-4. Specifications of used material amounts have been presented in Table (5).

Table 5. Materials used in the suspension for use in the electrophoretic process

\begin{tabular}{|c|c|c|}
\hline & Compound name & Amounts \\
\hline 1 & Isopropanol & $50 \mathrm{ml}$ \\
\hline 2 & Hydroxyapatite & $0.3 \mathrm{~g}$ \\
\hline 3 & Graphene oxide & $0.01 \mathrm{~g}$ \\
\hline 4 & Zirconia oxide & $0.02 \mathrm{~g}$ \\
\hline 5 & Triethanolamine & $2 \mathrm{~mL}$ \\
\hline
\end{tabular}

After preparing the suspension, to create the corresponding composite coating, the desired electrodes were first provided. Then, by plugging it into the power supply and putting it in the suspension solution at a specified voltage of $60 \mathrm{~V}$ and a predetermined duration of $180 \mathrm{~s}$, the coating was applied to the base. Consequently, to build strength and to increase the adhesion of the composite coating, sintering was used. To this end, the coated sample was set in a vacuum furnace oven for 2 hours at $950^{\circ} \mathrm{C}$ and then, cooled down in the furnace.

MAP analysis was adopted to review the distribution of additives. Elemental analysis was executed by EDAX EDS Silicon Drift 2017 detector. A Philips Xpert model with a current of $31 \mathrm{~mA}$ and a voltage of $41 \mathrm{kV}$ was employed to investigate the crystalline structure of the synthesized powders. The utilized cathode lamp was a copper with a wavelength of 1,4114 angstroms. A scan rate of 1 and a step size of $1.11 / \mathrm{s}$ were decided. The identification of the phases was conducted by X-Pert software.

Metal samples were prepared first to conduct electrochemical experiments on different samples in the desired solution. Before testing, all samples were degreased with alcohol and then rinsed with distilled water. After the preparation, each sample was installed on the cell as an operating electrode, and a counter was used to complete the circuit and do the test. Then, the solution inserted into the cell. The heating system, including a heating element and a thermometer, was used to adjust the temperature. For electrochemical measurements by electrochemical polarization spectroscopy, potentiostat-galvanostat IVIUMSTAT with IVIUMSOFT execution software was used.

Necessary preparations need to be carried out 24 hours before running the antibacterial test. For this purpose, the necessary growth medium were provided and they were placed in an autoclave for 15 minutes at $121^{\circ} \mathrm{C}$ to sterilize the media. Also, the sample was sterilized with control. To prepare the required bacteria, they were subcultured 24 hours before the test.

To run the test, the desired suspension was prepared from the subcultured colonies. To prepare the desired suspension at a concentration of $10^{8} \mathrm{CFU} / \mathrm{ml}$, it was necessary to compare it with half McFarland (To prepare this solution, add $0.05 \mathrm{ml}$ of $1 \%$ barium chloride $\left(\mathrm{Bacl}_{2}\right)$ solution to $0.95 \mathrm{ml}$ of $1 \%$ sulfuric acid $\left(\mathrm{H}_{2} \mathrm{SO}_{4}\right)$ solution and shake well). To accurately determine the number of bacteria by spectrophotometer, its absorption rate was measured. The needed absorbance for a concentration of $10^{8} \mathrm{CFU} / \mathrm{ml}$ was $625 \mathrm{~nm}$. Next, $1 \mathrm{cc}$ of the suspension with the desired dilution was added to the alloy facade (The pure titanium alloy was chosen as the control). Consequently, it was put in a $37^{\circ} \mathrm{C}$ incubator for 24 hours. Next, the bacteria was collected from the alloy surface by the sampler and they were them to the tube holding $10 \mathrm{ccs}$ of Ringer culture medium and vortex. Then, by 
diluting the concentrations of $10^{5}, 10^{6}$ and $10^{7}$, the culture was provided by the spread-plate method. The plates were placed in a 37-degree incubator after closing. In 24 hours, the number of colonies were calculated by the colony counter and their number were reported. Gram-positive bacteria (Escherichia coli) with ATTC29213 and gram-negative bacteria (Staphylococcus aureus) with ATTC25922 were used in this experiment.

\section{Findings}

The current study aimed to characterize the nanocomposite coating of hydroxyapatite, zirconia, and graphene oxide on titanium substrate using the polarization method. In the first step, while describing the composite coating formed by scanning electron microscopy, the thickness and uniformity of the coat were studied. In the second step, the X-ray diffraction test was used to study and plot the arrangement of the materials used in the coating. In the third step, electrochemical tests were investigated by chemical polarization to evaluate the corrosion behavior of the coating owing to the addition of nanoparticles to the hydroxyapatite and it was compared with the uncoated sample. The analysis was then carried out by drawing the corresponding charts. Finally, in the fourth step, antibacterial tests were performed on Escherichia coli and Staphylococcus aureus on the obtained coating, and the results were compared with non-coated alloy samples.

\section{Evaluation of coating structure and morphology}

To investigate the coating attributes, including coating surface morphology, coating thickness, the arrangement of coating elements, and coating's connection with the substrate, scanning electron microscopy images of the coating surface have been exhibited in Figure (2).
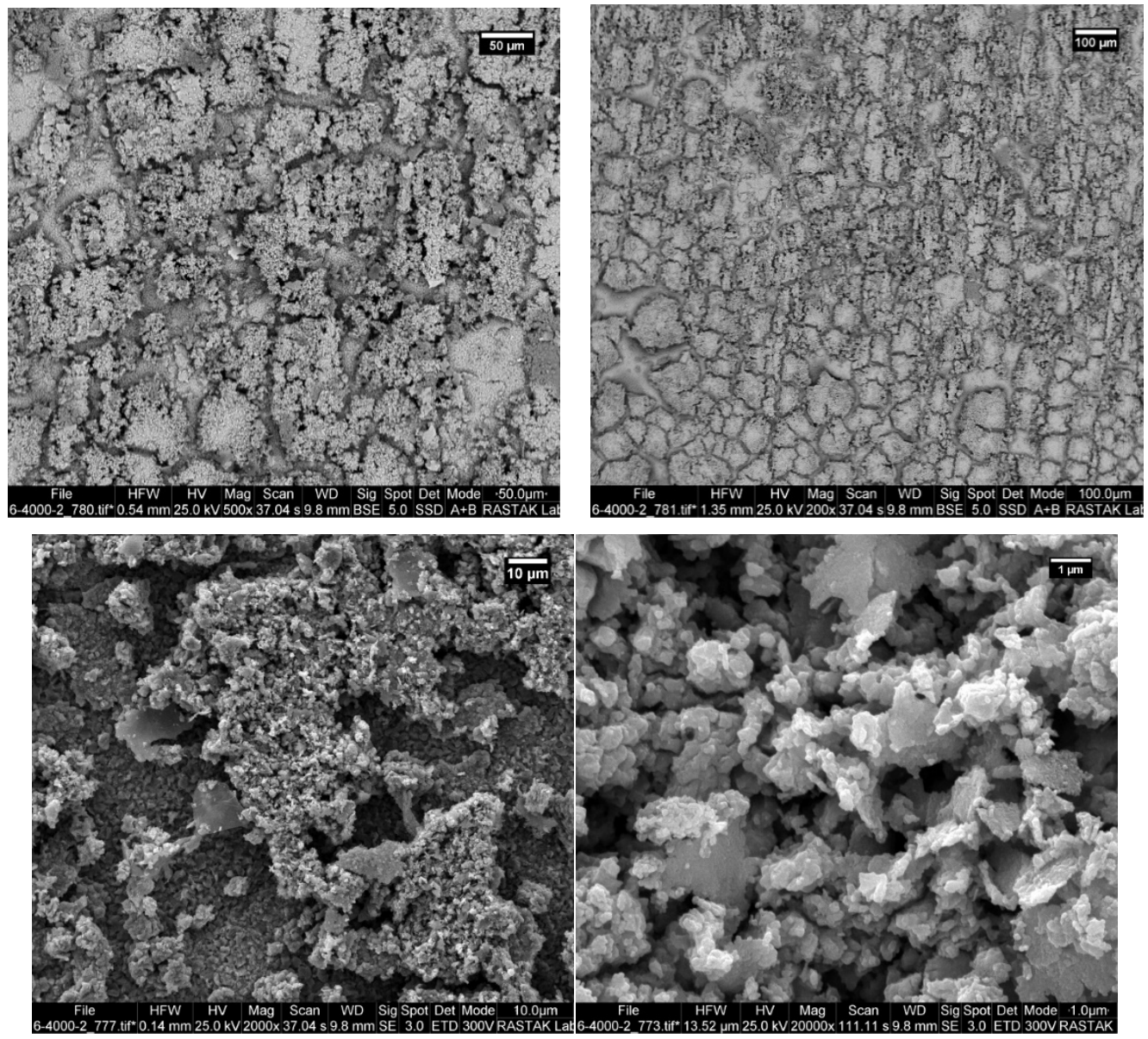

Figure 2. Scanning electron microscopy images of the surface of the coatings obtained at different magnifications.

This figure presents scanning electron microscopy images of the sample surface after synthesis in a vacuum furnace at $950^{\circ} \mathrm{C}$. Studies revealed that hydroxyapatite coatings displayed poor mechanical and antibacterial qualities. Moreover, one of the best methods to enhance the properties of hydroxide apatite coatings is to create coatings using a secondary phase that may have a more reliable performance compared to pure hydroxide apatite coatings. The uniform morphology and agglomeration of the powders employed in the images are evident. The results proved that employing the secondary phase created adhesion to the substrate and a crack-free as well as a high uniformity surface for the coating. 
In this regard, Arakovich et al. noted in a study named "Characterization of Hydroxyapatite / Chitosan / Graphene oxide coatings on a titanium layer using electrophoretic method" that after increasing the coating time from 5 minutes to 10 minutes, the morphology of the hydroxyapatite surface changed, and active hydroxyapatite particles started to be more engaged. This is what makes these particles more visible than before. Moreover, they recognized that after increasing the coating time from $10 \mathrm{~min}$ to $15 \mathrm{~min}$ at a constant $\mathrm{pH}$, the thickness of the hydroxyapatite coating increased [15]. Also, in an article entitled "Electrophoretic Method of Characterization and Coating of Hydroxyapatite Powder, Graphene Oxide and Chitosan", Shi et al. decided that the thickness and size of coatings increased with the addition of chitosan and graphene to hydroxyapatite [16]. Evaluation outcomes determined that the coating thickness increased with the increase of coating duration.

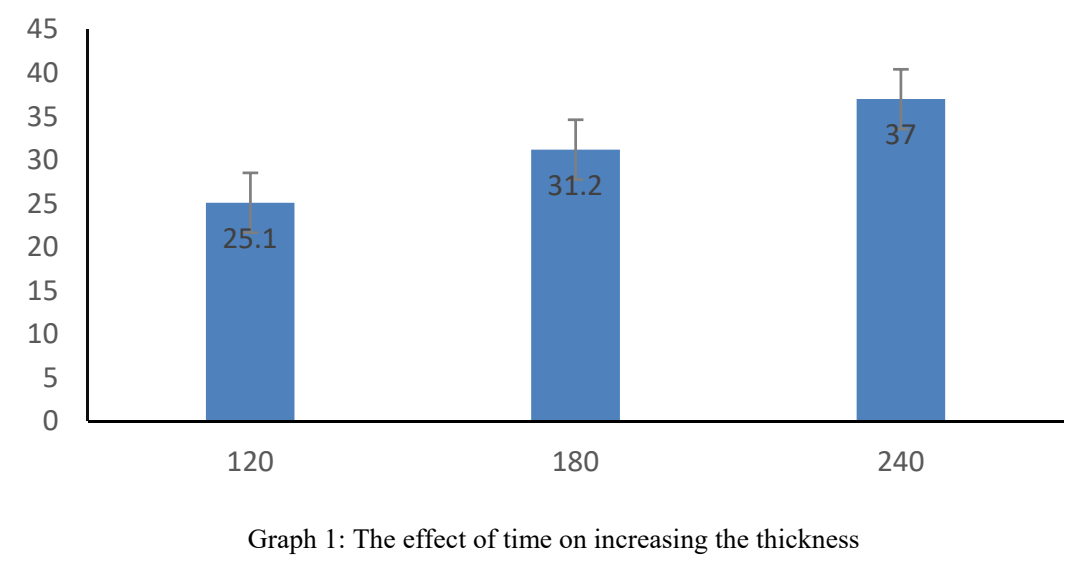

Figure (3) shows the results of the elemental EDAX analysis. Identification phase in the microstructure of the material by chemical composition plays an essential role in recognizing and determining the unknown phases. It is worth remarking that this method was intended for quantitative analysis of materials using scanning electron microscopy and was not a replacement for regular elemental analysis tests; rather it was used as an appropriate standard method, especially for achieving dative chemical composition and quantitative and qualitative investigation of phases.

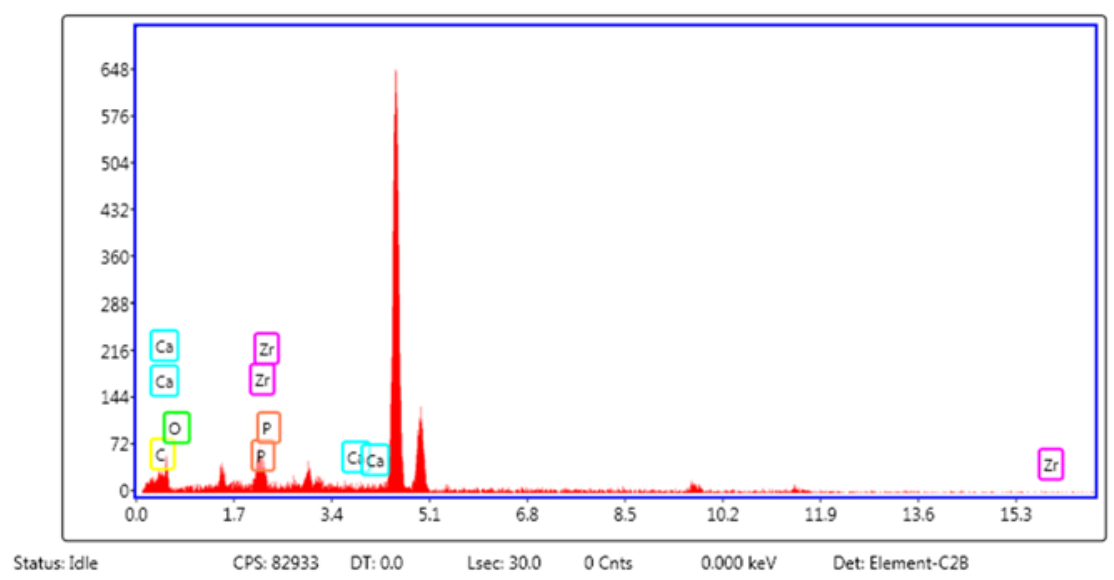

Figure 3: Elemental analysis image of EDAX hydroxyapatite-graphene oxide-zirconia composite

As displayed in Figure 4, the hydroxyapatite/graphene oxide/zirconia oxide composite coating was entirely coated on the surface. In this study, graphene oxide was added as a reinforcer to the composite composition, which is evident in black. 


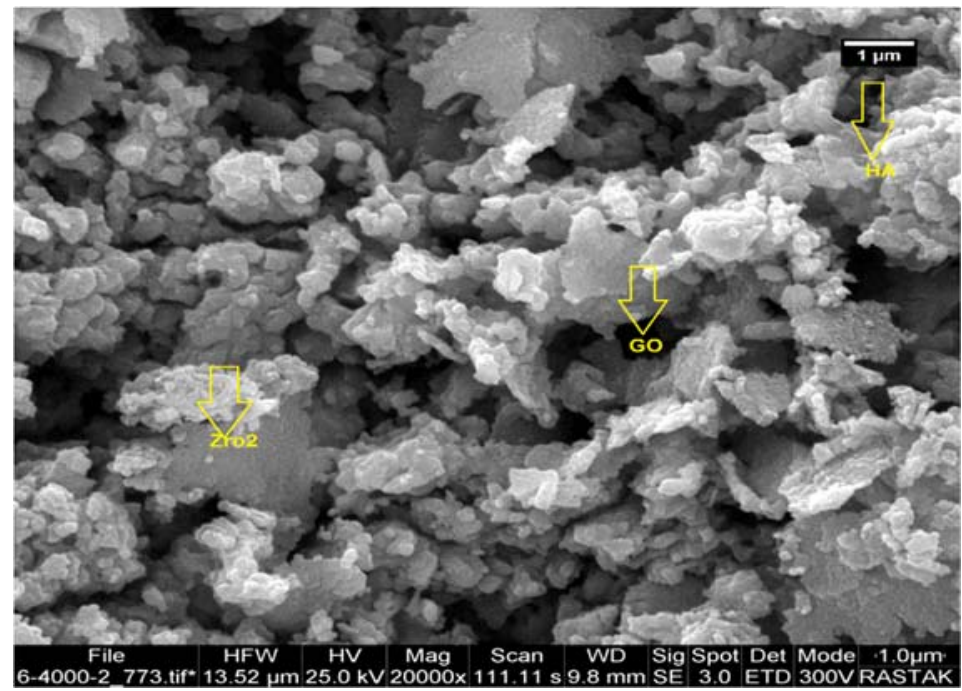

Figure 4: Distribution of the elements present on the composite coat

A) gray area: hydroxyapatite; b) light area: zirconia oxide; and c) dark area: graphene oxide

Figure (5) presents the MAP analysis for all elements present in the composite coating. The distribution of elements such as carbon, oxygen, calcium, zirconium, oxygen, and phosphorus has been exhibited in this figure. These images designated that given the values adopted in the laboratory, the elements were coated accurately on the surface.
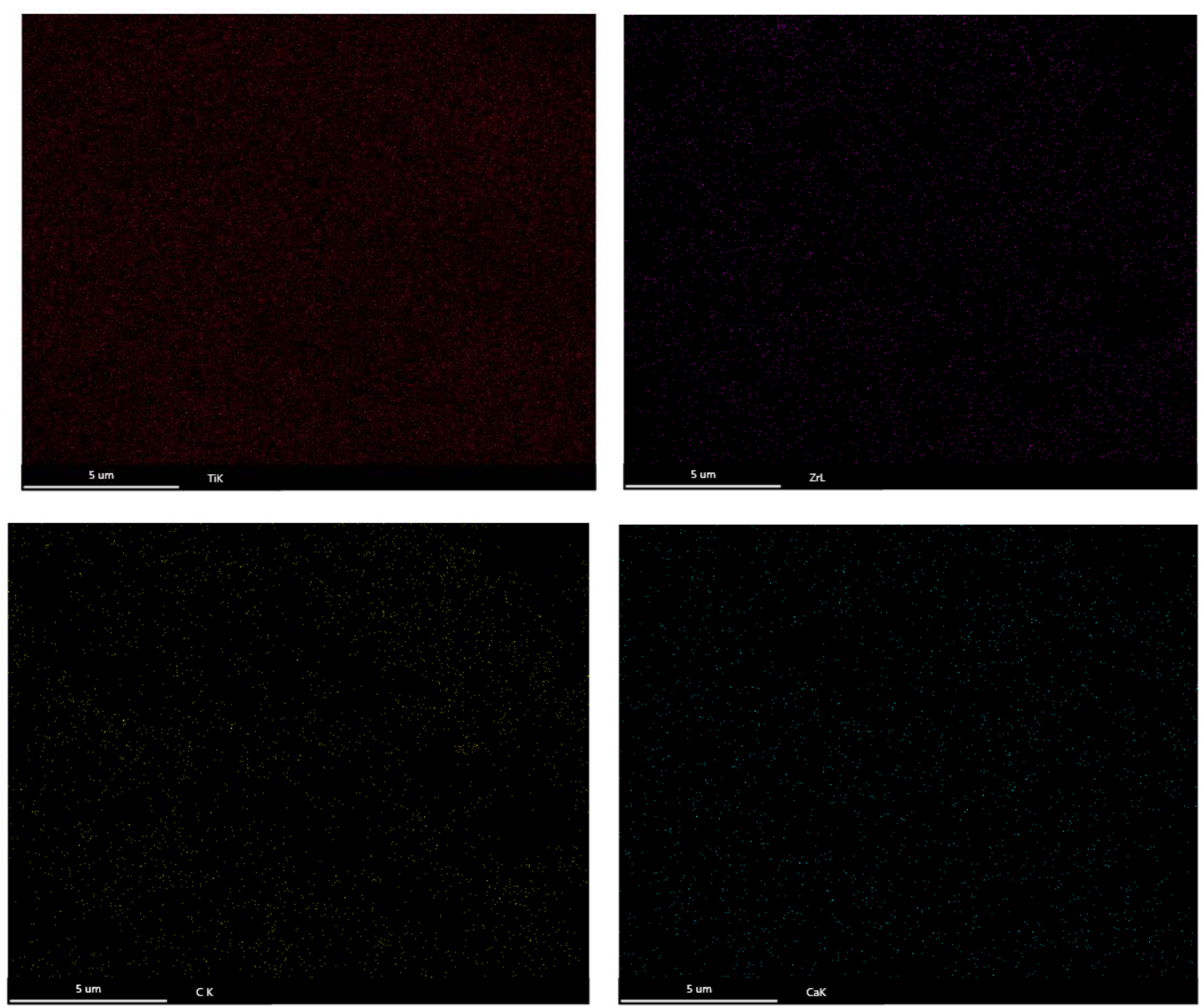

Figure 5: MAP image of all the elements present in the composite coating 


\section{Fuzzy analysis}

X-ray diffraction device was used for (structural) finding the phases of the coatings. In this regard, in a study entitled "Synthesis of Hydroxyapatite / Copper and Hydroxyapatite / Oxide Nano-Composite Coatings by Electrophoretic Method and Analysis of Corrosion Resistance of Coatings formed in Simulated Body Solution", through analyzing and investigating the phases of the study, Rafieinia et al. found that there was no other calcium phosphate phases except the hydroxyapatite in the structure. In addition, the results of the X-ray diffraction peaks confirmed the presence of the peaks that were corresponding to the crystalline plates (211), (002), (112), (202) [17].

By analyzing the phases and comparing the X-ray diffraction pattern with the literature, it was concluded that all peaks of the composite elements of hydroxyapatite, zirconium oxide, graphene oxide are observable. A comparison of the peaks achieved with standard JCPDS cards revealed that all the phases on the coating were present in the XRD spectrum. These elements include Calcium, Phosphorus; Oxygen; Carbon, Zirconium Oxide and Titanium.

\section{Evaluation of corrosion resistance of the coating}

The corrosion resistance of the created coatings was determined by the electrochemical polarization test of a threeelectrode cell comprising of saturated calomel, and platinum electrodes as the reference and counter electrodes, and coated samples as working electrodes. The polarization test was further conducted in simulated body solution at $37^{\circ} \mathrm{C}$.

The results of the polarization test proved that the utilized coatings enhanced the substrate's corrosion resistance. The $10 \% \mathrm{CuO}$ hydroxyapatite coating had the highest corrosion resistance, while the $10 \%$ copper hydroxyapatite had the least corrosion resistance.

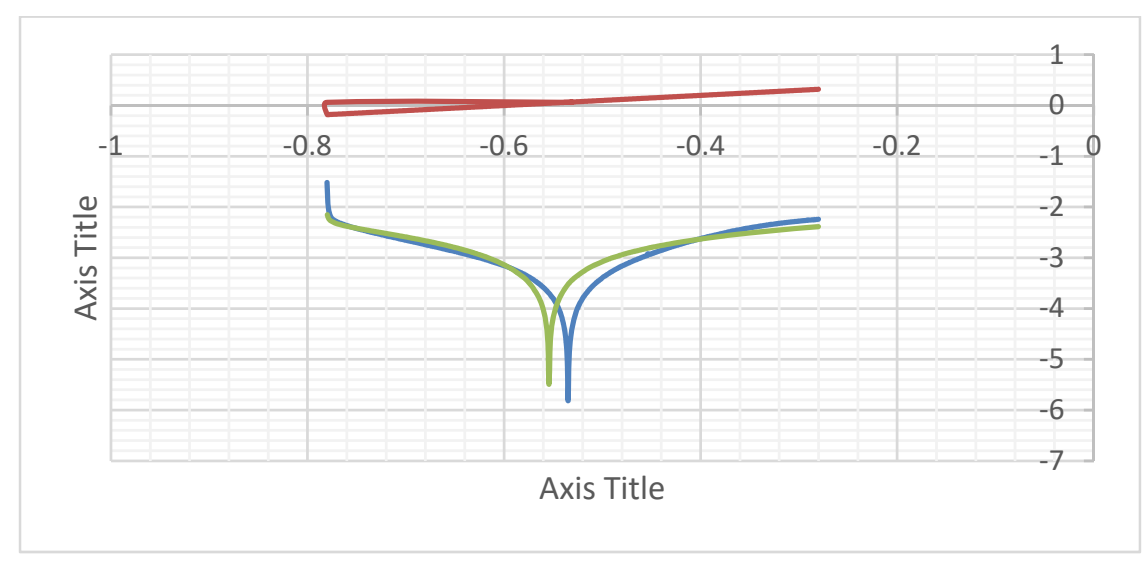

Figure 6: Curve diagram of the electrochemical polarization test

A) graph of Ti6AL4V alloy (green), b) the coated sample (blue)

By analyzing Figure 6, it was discovered that the graph moved toward zero, and the negative potential of corrosion resistance decrement increased. Therefore, it can be settled that the coated sample attained higher corrosion resistance in the electrochemical polarization test compared to the uncoated sample, which was proved by inspecting the provided graph.

In a study named "Improving the Corrosion and Adhesion Behavior of Biocrystalline Glass Coating on Pure Commercial Titanium Using a Combination of Microarc Oxidation and Electrophoretic Coating Layers Polarization Measurement in Body Simulation Solution" Farnoosh proved that using bioglass coating on the adjusted titanium substrate by electrophoretic method enhanced the corrosion resistance and adhesion of the coating to the substrate [18].

\section{Evaluation of antibacterial behavior}

In the conducted tests, the hydroxyapatite coating presented a very approving dependence on the bacterial adhesion. Consequently, it is required to supplement new substances or compounds to hydroxyapatite to develop the antibacterial property. By researching and adopting other researchers' papers, it was concluded that zirconia ceramic can be added to the hydroxyapatite/graphene oxide composite. As zirconium oxide has a very high-grade antibacterial activity, the nano oxide was added to the composite compound of hydroxyapatite/graphene oxide. In a study titled "Coating and Characterization of Hydroxyapatite / Graphene Oxide Nanocomposite by Electrophoretic Method", Ming et al. [19] settled that the coating produced against both gram-positive Escherichia coli and gram-negative Staphylococcus aureus is unprofitable, and has no impacts on decreasing the bacterial count and forming antibacterial activity. In this study, considering the results of the previous study, the antibacterial properties of both gram-positive (Escherichia) and gram-negative (Staphylococcus) bacteria were 
examined. It was observed that antibacterial property was significantly increased in the hydroxyapatite, zirconia oxide, and graphene oxide combination. The initial value of both Gram-positive bacteria (Escherichia) and Gramnegative bacteria (Staphylococcus) was $5 * 10^{7} \mathrm{CFU} / \mathrm{ml}$. After 48 hours, it was noted that the number of bacteria had declined by an average of 1.2 logarithms, which was significantly more reliable than that of previous studies. This hints that adding zirconium oxide to the composite coating could enhance the sought antibacterial properties.

Also, after counting, the number of $1.2410^{6} \mathrm{CFU} / \mathrm{ml}$ was obtained as shown in Figure (7), which displays the number of Escherichia coli and it was concluded that the number of bacteria was significantly reduced.

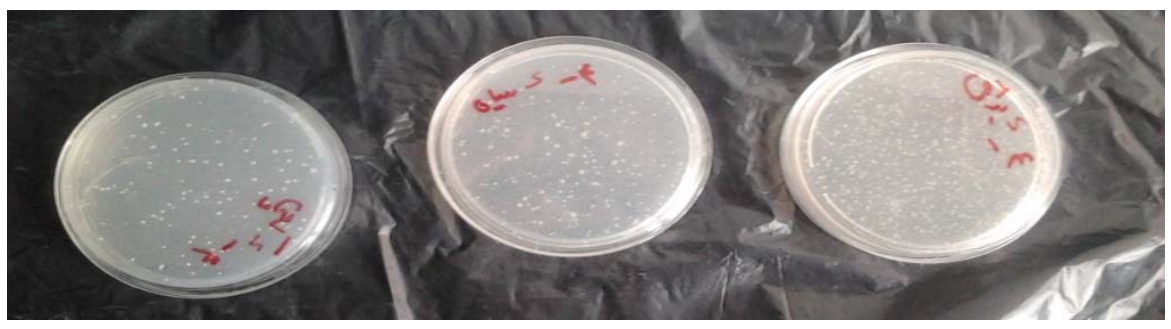

Figure 7: Escherichia coli pictures

\section{Conclusion}

1-Scanning electron microscopy images proved that the cross-section of the hydroxyapatite/zirconium oxide/graphene oxide composite coating had a thickness of about 22 to $26.5 \mu \mathrm{m}$.

2- Scanning electron microscopy images of the coating surface revealed that the microstructure of the coatings was uniform and free from microscopic cracks.

3- The results of the electrochemical polarization test confirmed that the corrosion resistance of the composite coating formed was much more reliable than that of the uncoated substrate.

4. Microbial research showed that the antibacterial activity of the hydroxyapatite/zirconium oxide and graphene oxide composites had increased significantly in comparison to the uncoated samples.

\section{References}

[1] Park Joon, R.S. Lakes. Biomaterials: an introduction, third ed : springer media ;2007.

[2] Hench larry. An introduction to bioceramics, 2 nd ed.: imperial college press;2013.

[3] Gurappa I.. Characterization of different materials for corrosion resistance under simulated body fluid conditions. Materials characterization $2002 ; 49: 73-79$

[4] Muresan L.M... Corrosion protective coatings for Ti and Ti alloys used for biomedical implants. James Waney. Intelligent coatings for corrosion:2014. Pp .585-602

[5] par; joon. Bio ceramics: properties , characterization, and application. Springer science business media; 2008.

[6] kokubo tadashi. Bio ceramics and their clinical application. : woodhead publishing limited;2008.

[7] K.M. Lee, B.U. Lee, S. Yoon, E.S. Lee, Y. Bongyoung, D.H. Shin, Electrochim. Acta 67 (2012) 6-11.

[8] B.R. Sunil, A.A. Kumar, T.S. Sampathkumar, C. Uday, Mater. Sci. Eng. C 33 (2013) 1607-1615.

[9] Chen, D., Tang, L., Li, J., "Graphene-Based Materials in Electrochemistry", Chemical Soceity Reveiw, Vol.39, pp.3157-3180 (2010).

[10] J. Berdy, Bioactive microbial metabolites, J. Antibiot. 58 (2005) 1-26.

[11] Ming LI,Pan XIONG, Maosong MO, Yan CHENG, and Yufeng ZHENG, Electrophoretic-deposited novel ternary silk fibroin/graphene oxide/hydroxyapatite nanocomposite coatings on titanium substrate for orthopedic applications, Higher Education Press and SpringerVerlag Berlin Heidelberg 2016.

[12] Ana Janković, Sanja Eraković, Miodrag Mitrić, Ivana Z. Matić, Zorica D. Juranić, Gary C.P. Tsui, Chak-yin Tang, Vesna MiškovićStanković, Kyong Yop Rhee, Soo Jin Park, Bioactive Hydroxyapatite /Graphene Composite Coating and Its Corrosion Stability in Simulated Body Fluid. Bioactive Hydroxyapatite /Graphene Composite Coating and Its Corrosion Stability in Simulated Body Fluid,2014.

[13] Hamidreza Farnoush, Zohreh Rezaei, Effect of suspension stability on bonding strength and electrochemical behavior of electrophoretically deposited HA-YSZ nanostructured composite coatings , ceramic international,2017

[14] Ming Li, Pan Xiong, Feng Yan, Sijie Li, Changhong Ren, Zhichen Yin, Ang Li Huafang Li Xunming Ji, Yufeng Zheng, Yan Cheng, An overview of graphene-based hydroxyapatite composites for orthopedic applications.bioactive material.2017.

[15] Marija Đoši, Sanja Erakovi, Ana Jankovi, Maja Vukašinovi -Sekuli, Ivana Z. Mati_, Jovica Stojanovi_, Kyong Yop Rhee, Vesna Miškovi-Stankovi, Soo-Jin Parke, In vitro investigation of electrophoretically deposited bioactive hydroxyapatite/chitosan coatings reinforced by graphene, industrial biomaterial,2016.

[16] Y. Y. Shi M. Li Q. LiuZ. J. Jia1 X. C. Xu• Y. Cheng Y. F. Zhen, Electrophoretic deposition of graphene oxide reinforced chitosanhydroxyapatite nanocomposite coatings on Ti substrate, J Mater Sci: Mater Med (2016) 27:48.

[17] Hadidi, M., Rafieinia, M., Saebnorri, E. (2016). The synthesis of nanocomposites of hydroxyapatite/ Copper, hydroxyapatite/copper oxide with the electrophoretic method, and the study of resistance against corrosion of coatings in the simulated body fluid. Journal of advanced materials and technologies;

[18] Farnoosh, H. (2016). The improvement of corrosion behavior and adhesion of active bioglass on pure titanium using the combination of micro-arc oxidation methods and electrophoretic spinning. Journal of advanced materials and technologies.

[19] Ming Li, Qian Liu, Zhaojun Jia, Xuchen Xu, Yan Cheng, Yufeng Zheng Tingfei Xi, Shicheng Wei, Graphene oxide/hydroxyapatite composite coatings fabricated by electrophoretic nanotechnology for biological applications,carbon journal, 2013. 